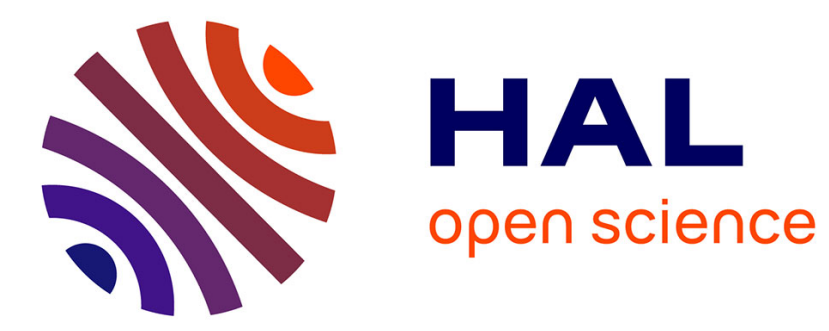

\title{
Core-level XPS spectra of supported 3d-metal ultrathin layers: experimental and theoretical studies
}

\author{
G. Garreau, V. Schorsch, E. Beaurepaire, J. Parlebas, O. Speder, P. Rennert
}

\section{To cite this version:}

G. Garreau, V. Schorsch, E. Beaurepaire, J. Parlebas, O. Speder, et al.. Core-level XPS spectra of supported 3d-metal ultrathin layers: experimental and theoretical studies. Journal de Physique IV Proceedings, 1994, 04 (C9), pp.C9-127-C9-130. 10.1051/jp4:1994918 . jpa-00253478

\section{HAL Id: jpa-00253478 https://hal.science/jpa-00253478}

Submitted on 1 Jan 1994

HAL is a multi-disciplinary open access archive for the deposit and dissemination of scientific research documents, whether they are published or not. The documents may come from teaching and research institutions in France or abroad, or from public or private research centers.
L'archive ouverte pluridisciplinaire HAL, est destinée au dépôt et à la diffusion de documents scientifiques de niveau recherche, publiés ou non, émanant des établissements d'enseignement et de recherche français ou étrangers, des laboratoires publics ou privés. 


\title{
Core-level XPS spectra of supported 3d-metal ultrathin layers: experimental and theoretical studies
}

\author{
G. Garreau, V. Schorsch, E. Beaurepaire, J.C. Parlebas, O. Speder* and P, Rennert* \\ IPCMS-GEMME, UM 46 du CNRS, 23 rue du Loess, BP. 20 CR, 67037 Strasbourg, France \\ * Dept. of Physics, Martin Luther Univ., Friedeman Bach Pl. 6, 06099 Halle, Germany
}

\begin{abstract}
In order to discuss the relation between the core level c-XPS spectra and the local electronic and magnetic properties of supported 3d transition metal atoms, we incorporate the various Coulomb and exchange interactions into a generalized impurity Anderson Hamiltonian, both in the initial state $\left(\mathrm{U}_{\mathrm{dd}}, \mathrm{J}_{\mathrm{dd}}\right)$ and final state $\left(\mathrm{U}_{\mathrm{dc}}, \mathrm{J}_{\mathrm{dc}}\right)$ of the c-XPS process. Then we discuss recent experimental $3 \mathrm{~s}$-XPS spectra of $\mathrm{Fe}$ and $\mathrm{Co}$ ultrathin-layers upon $\mathrm{Cr}(100)$ as well as $\mathrm{V}$ clusters upon graphite in terms of the two limits of the preceding Hamiltonian : (1) the exchange splitting limit $\left(\mathrm{J}_{\mathrm{dc}}\right)$ and (2) the dielectric relaxation limit $\left(\mathrm{U}_{\mathrm{dc}}\right)$.
\end{abstract}

\section{INTRODUCTION}

Quite recently there has been a lot of spectroscopic measurements in order to test the appearance of magnetism on supported 3d-metal ultrathin-layers. For example, inverse photoemission spectroscopy [1] as well as electron capture spectroscopy [2] pointed out that an overlayer of $\mathrm{V}$ on $\mathrm{Ag}(100)$ is ferromagnetic, whereas spin-polarized X-ray Photoemission Spectroscopy (XPS) [3] and surface magnetooptical Kerr effect experiments [4] showed no such evidence for magnetism. Nevertheless, contamination could be a possible source of disagreement between the previously considered experiments. As for $\mathrm{V}$ on graphite, Binns et al. [5] pointed out recently that the appearance of magnetic moments on $\mathrm{V}$ can be quenched after exposure to $\mathrm{CO}$ pollutant ; only for freshly evaporated V clusters on graphite, these authors found a satellite structure in the V $3 \mathrm{~s}$ core-level XPS which they attributed to the presence of magnetic moments on the $\mathrm{V}$ adatoms. In the present work we study and discuss the various origins of such satellite structures in core-level XPS spectra (c-XPS).

\section{GENERAL MODEL HAMILTONIAN FOR CORE-LEVEL XPS SPECTRA}

Core-level XPS spectra reflect strongly the many-body effects arising from the outer electrons of the irradiated material $[6,7]$. When a core hole is produced by the incident photon, the outer electron system is perturbed by the core-hole charge and screens it. Thus the core hole plays a role of 'test charge' which induces the many-body (dielectric) response of the outer electron system. As the core hole has not only a charge but also a spin, it can also play the role of a 'test spin' to induce a magnetic response from the outer electron system. Before looking into both aspects in more details, let us first express a possible general Hamiltonian for c-XPS spectra when a given $3 \mathrm{~d}$ metal atom, adsorbed on a substrate, is irradiated. In the ground state, i.e. before irradiation, the generalized 'impurity' Anderson Hamiltonian is written as : 


$$
\begin{aligned}
& H_{g}=\sum_{n k \sigma} \epsilon_{k}^{n \sigma} c_{k}^{+n \sigma} c_{k}^{n \sigma}+\sum_{m \sigma} \epsilon_{A}^{m \sigma} a^{+m \sigma} a^{m \sigma}+\sum_{\operatorname{lmR} \sigma} V_{R A}^{I m}\left(c_{R}^{+l \sigma} a^{m \sigma}+h . c .\right)+U^{d d} \sum_{m m^{\prime}} a^{+m \uparrow} a^{m \uparrow} a^{+m^{\prime} \downarrow} a^{m^{\prime} \downarrow} \\
& +\frac{1}{2}\left(U^{d d}-J^{d d}\right) \sum_{\substack{m m^{\prime} \sigma \\
\left(m \neq m^{\prime}\right)}} a^{+m \sigma} a^{m \sigma} a^{+m^{\prime} \sigma} a^{m^{\prime} \sigma}
\end{aligned}
$$

In eq. (2.1) $\left\{\varepsilon_{\mathrm{k}}^{\mathrm{m} \sigma}\right\}$ are the eigenenergies of the substrate surface eigenstates $\mid$ nko $\rangle$ with wave number $k$ and spin $\sigma ;\left\{c_{k}^{+n \sigma}\right\}$ being the corresponding creation operators [8]. The operator $a^{+m \sigma}$ creates a state |Amo) at adsorption site $A$, with $m$ symmetry $\left(m=s, x y, y z, z x, x^{2}-y^{2}, 3 z^{2}-r^{2}\right), s p i n \sigma$ and energy $\epsilon_{A}^{m \sigma}$. For example in the case of a hollow position $A$ on graphite, the summation on $R$ in eq. (2.1) is restricted to the 6 first neighbours of the hexagonal ring below $A$ [9]. V $V_{R A}^{\operatorname{lm}}$ labels the hybridization between $|A m \sigma\rangle$ and $|R l \sigma\rangle$. Finally $U^{d d}$ and Jdd are, respectively, the Coulomb and exchange interactions between 3d electrons [9] : Hartree-Fock magnetic solutions have been worked out for vanadium and chromium upon graphite and for reasonable Jdd values. Then, in the final state of the c-XPS process, a core-hole is created at site $\mathrm{A}$ by an incident photon and the final Hamiltonian :

$$
H_{f}=H_{g}-U^{d c} \sum_{m \sigma} a^{+m \sigma} a^{m \sigma}-2 J^{d c} \vec{S} \cdot \vec{s}
$$

where $U^{d c}$ represents the Coulomb interaction between $3 \mathrm{~d}$ electrons of $\mathrm{A}$ and the core-hole potential, whereas $\mathrm{Jdc}$ is the exchange interaction which couples the hole spin $\overrightarrow{\mathrm{s}}$ and a $3 \mathrm{~d}$ spin $\overrightarrow{\mathrm{S}}$ at site $\mathrm{A}$.

\section{EXCHANGE SPLITTING EFFECT DUE TO THE CORE HOLE SPIN}

In this section we only keep the exchange coupling term $\mathrm{Jdc}^{\mathrm{dc}}$, i.e. we ignore $\mathrm{U}^{\mathrm{dc}}$ in eq. (2.2). Moreover, for simplicity, we will also ignore the hybridization of eq. (2.1), recovering a purely atomic $\mathrm{Jdc}$ model in the following interpretations. As typical experimental spectra, we show in figs la and $b$, respectively the $\mathrm{Fe}$ and Co 3 s XPS of epitaxial ultrathin layers deposited on a $\mathrm{Cr}(100)$ surface $[10,11]$ in comparison to bulk Fe and Co $3 \mathrm{~s}$ XPS. In the case of bulk Fe $3 \mathrm{~s}$ XPS, it is generally assumed that the exchange interaction between the core hole spin s and the $3 \mathrm{~d}$ electron spin $S$ gives rise to a satellite on the high binding energy side of the main line of the spectrum [12]. If this is true for the bulk, it should be also true for ultrathin layers. At least, the magnetic multiplet is as much evidenced (down to the lowest Fe coverage) as in the bulk Fe case (Fig. la), suggesting that the Fe local moment is unchanged $( \pm 10 \%)$ when going from bulk to ultrathin layers on $\operatorname{Cr}(100)$. Moreover in the case of a $2.5 \AA$ Co layer, the satellite is even more pronounced than in the bulk Co case (Fig. 1b).

Within the above mentioned Jdc model, the projected states $S z=\{-S, \ldots+S\}$ are degenerate in the initial state of the 3s-XPS. In the final state, we obtain the multiplets with the total spin $S_{\text {tot }}=|S+s|=\left\{S+\frac{1}{2} ; S-\frac{1}{2}\right\}$, the energies of which components are $-J d c S$ and $J d c(S+1)$, respectively. The corresponding exchange split $3 \mathrm{~s}$ XPS spectrum is given by $[6,7]$ :

$F_{e x}\left(E_{B}\right)=\frac{S+1}{2 S+1} \delta\left(E_{B}+J^{d c} S\right)+\frac{S}{2 S+1} \delta\left(E_{B}-J^{d c}(S+1)\right)$

Thus it is well known that the $3 \mathrm{~s}$ XPS splits into two peaks with the intensity ratio $I_{R}=(S+1) / S$ and the energy separation $\Delta \mathrm{E}_{\mathrm{B}}=\mathrm{J}^{\mathrm{dc}}(2 \mathrm{~S}+1)$. For example, if we estimate $1.2 \leq \mathrm{I}_{\mathrm{R}} \leq 2$ (Fig. 1a), we obtain $1 \leq \mathrm{S} \cong\left(\mathrm{I}_{\mathrm{R}}-\mathrm{l}\right)^{-1} \leq 5$ for $\mathrm{Fe}$; from $\Delta \mathrm{E}_{\mathrm{B}} \cong 4,5 \mathrm{eV}$ (Fig. 1a) we can also deduce $0.4 \mathrm{eV} \leq \mathrm{J}^{\mathrm{dc}} \leq 1.5 \mathrm{eV}$ for $\mathrm{Fe}$ (for a comparison to the values $J_{H F}^{d c}$ see $[6,7]$ ). Similarly if we assume $I_{R} \cong 3$ (Fig. $1 \mathrm{~b}$ ) we find $\mathrm{S}=1 / 2$ for $\mathrm{Co}$ and $\Delta \mathrm{E}_{\mathrm{B}} \cong 4.8 \mathrm{eV}$ yields $\mathrm{J}^{\mathrm{dc}} \cong 0.7 \mathrm{eV}$. From an experimental point of view the decomposition of the c-XPS spectra into a main line and a satellite is not unique and the intensity ratio $I_{R}$ is not well defined at all [13]. Moreover $I_{R}$ does not necessarily correlate with the magnetic moment since $3 \mathrm{~s}$ splittings were observed in compounds which do not have a magnetic moment $[14,15]$. 

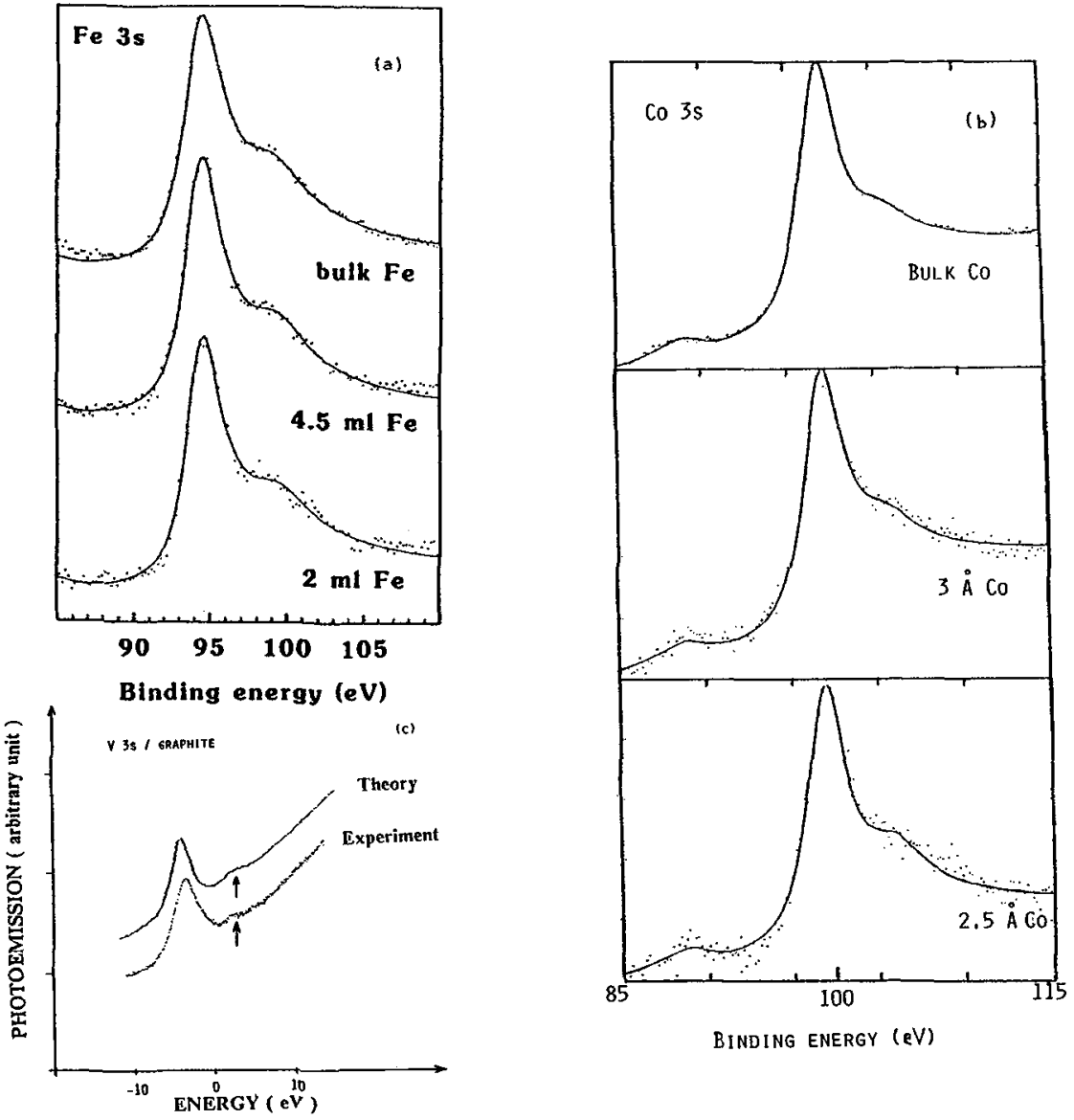

Fig. 1 - Experimental Fe (a) and Co (b) 3s XPS for bulk Fe and Co as well as two differing epitaxial thin Fe and Co films upon $\mathrm{Cr}(100)$; lines ate fits using two Doniach-Sunjic functions. Theoretical c-XPS spectrum (c) augmented by a parabolic background in comparison to the measured 3s-XPS of $\mathrm{V}$ upon graphite [5]

\section{DIELECTRIC RELAXATION EFFECT DUE TO THE CORE HOLE CHARGE}

If we apply the preceding atomic results of eq. (3.1) to the $3 \mathrm{~s} V$ XPS spectra of adsorbed vanadium upon graphite [5], which exhibits a much smaller satellite intensity, we obtain quite strange results $\left(\mathrm{S} \leq 1 / 3 ; \mathrm{J}^{\mathrm{dc}} \geq 3 \mathrm{eV}\right)$, suggesting that the $\mathrm{U}^{\mathrm{dc}}$ term of eq. (2.2) as well as the solid state effects of eq. (2.1), especially hybridization $V_{\mathrm{RA}}^{\mathrm{lm}}$, should now be taken into account. Actually, in this section and for simplicity, we ignore $\mathrm{Jdc}^{\mathrm{dc}}$ in eq. (2.2) and $\mathrm{Jdd}$ in eq. (2.1). We represent the graphite band structure, restricted to $\pi$-symmetry, by a completely filled ligand band (LB). This band is discrete, rectangular in shape and has a width of $W=5 \mathrm{eV}$. The spin-degenerate ' $3 \mathrm{~d}$ ' level $\epsilon_{A}^{\mathrm{mo}} \equiv \epsilon_{\mathrm{A}}=2.5 \mathrm{eV}$ is located just at the top of the filled $\mathrm{LB}$; the centre of $\mathrm{LB}, \epsilon_{\mathrm{L}}^{\mathrm{o}}$, being the energy origin. Due to configuration interaction, in the ground state of the $3 \mathrm{~s}$-XPS, we have to consider a strong mixture of $3 d^{\circ}$ and $3 d^{1} \underline{L}$ configurations, where $\underline{L}$ means a ligand hole in the LB ; moreover, because $U_{d d}$ is finite in eq. (2.1) we also consider the configuration $3 \mathrm{~d}^{2} \mathrm{~L}^{2}$ in the initial state of the $3 s$-XPS process. Then, in the final state, the $3 \mathrm{~d}$ level of vanadium is pulled down by the core hole potential $-\mathrm{U}^{\mathrm{dc}}$ and a similar configuration interaction mixture takes place between $\underline{c} 3 \underline{d}^{1} \underline{L}$ and $c 3 d^{2} \underline{L}^{2}$ where $\underline{c}$ labels the 3 s core hole $[6,7]$. The charge relaxed $3 \mathrm{~s}-\mathrm{XPS}$ spectrum is finally expressed as :

$F_{\text {ch }}\left(E_{B}\right)=\sum_{f}|\langle f \mid g\rangle|^{2}(\Gamma / \pi) /\left[\left(E_{B}+E_{g}-E_{f}\right)^{2}+\Gamma^{2}\right]$ 
where the $|f\rangle$ 's are all the possible final states for the electronic system ; $\Gamma$ labels the spectral broadening of an empirical Lorentzian function arising from the finite lifetime of the core hole as well as the experimental resolution width $(\Gamma=\mathrm{leV}$ in this paper). However within the present filled band model, the 3s-XPS spectrum of eq. (4.1) can be calculated exactly [7] : hybridization V (an average of $V_{R A}^{\mathrm{lm}}$ ) has a direct effect on the existence and the position of the satellite with respect to the main line whereas $U^{d c}$ must be strong enough $\left(U^{\mathrm{dc}} \geq 3 \mathrm{eV}\right.$ ) to give rise to a distinct satellite. Finally we compare (fig. 1c) our calculated result for $\mathrm{U}^{\mathrm{dc}}=4 \mathrm{eV}, \mathrm{U}^{\mathrm{dd}}=3 \mathrm{eV}, \Delta=2.5 \mathrm{eV}$ and $\mathrm{V}=2.5 \mathrm{eV}$, to the experimental spectrum $3 \mathrm{~s}$ XPS for vanadium adsorbed upon graphite [5].

\section{CONCLUSION}

In this short paper we discussed the core-level (especially 3s) XPS spectra of supported 3d metal ultrathin layers (and clusters) in terms of the possible detection of local magnetism. Our conclusion is twofolds. From a theoretical point of view it is necessary to extent the resolution of the 'impurity' Anderson model $(2.1-2.2)$ by taking into account both $\mathrm{U}^{\mathrm{dc}}$ and Jdc $[16,17]$ as well as the orbital degeneracy of the d level $[7,9,18]$. From an experimental view point, nothing can be inferred definitively about the coupling $\mathrm{Jdc}$ between magnetic moments in the absence of spin resolved data [19]. For example, in the ferromagnetic state of adsorbed transition metals, spin-polarized 3s XPS experiments should be able to distinguish multiplet peaks from peaks due to charge fluctuations. Moreover in the case of supported metallic clusters, angle resolved XPS would discriminate between surface-type and bulk-type metallic-atom contributions.

\section{Acknowledgements}

One of the authors (JCP) would like to acknowledge fruitful discussion and collaboration with

A. Kotani, C. Demangeat and A. Rakotomahevitra.

\section{References}

[1] Drube W and Himpsel FJ Phys. Rev. B35 (1987) 4131.

[2] Rau C, Xing G and Robert M J. Vac. Sci. Technol. A6 (1988) 579.

[3] Stamponi M, Vaterlaus A, Pescia D, Aeschlimann M, Meier F, Dürr W and Blügel S Phys. Rev. 37 (1988) 10380.

[4] Fink RL, Ballentine CA, Erskine JL and Araya-Pochet JA Phys. Rev. B41 (1990) 10175.

[5] Binns C, Derbyshire HS, Bayliss SC and Norris C Phys. Rev. B45 (1992) 460.

[6] Kotani A in 'Handbook on Synchrotron Radiation' GV Marr Ed (Elsevier Science Publishers B.V.) 2 (1987) 611.

[7] Kotani A, Jo T and Parlebas JC Advances in Phys. 37 (1988) 37

[8] Rakotomahevitra A, Garreau G, Demangeat C and Parlebas JC Surface Science (1994).

[9] Rakotomahevitra A, Demangeat C and Parlebas JC J. of Phys. : Condens. Matter (1994).

[10] Beaurepaire E, Scheurer F, Schorsch V, Carrière B, Deville JP and Heckmann O Surface Science 251/252 (1991) 36.

[11] Schorsch V, Rap. Stage DEA Strasbourg (1990).

[12] Fadley CS and Shirley DA Phys. Rev. A2 (1970) 1109.

[13] Hillebrecht F.U., Jungblut R and Kisker E Phys. Rev. Let. 65 (1990) 2450.

[14] Van Acker JF, Stadnik ZM, Fuggle JC, Hoekstra HJ, Buschow KH and Stroink G Phys. Rev. B 37 (1988) 6827.

[15] Oh SJ, Gweon GH and Park JG Phys. Rev. Let. 68 (1992) 2850 and Phys. Rev. B48 (1993) 7825.

[16] Kakehashi Y and Kotani A Phys. Rev. B29 (1984) 4292.

[17] Kakehashi Y and Fulde P Phys. Rev. B32 (1985) 1595.

[18] Parlebas J C, Beaurepaire E, Ikeda T and Kotani A J. Phys. France 51 (1990) 639.

[19] Van Campen DG and Klebanoff LE Phys. Rev. B49 (1994) 2040. 